\title{
Fault diagnosis of Steam Turbine Generator Unit Based on Support Vector Machine
}

\author{
Lin Sang ${ }^{1}$ and Tieshan Zhang ${ }^{2}$ \\ ${ }^{1}$ Institute of Electrical and Information Engineering, \\ Heilongjiang University of Science and Technology, Harbin, China \\ ${ }^{2}$ Institute of Electrical Power and Information Engineering, \\ China University of Mining and Technology Yinchuan College, Yinchuan, China
}

Sanglin198282@163.com,zts336699@126.com

\begin{abstract}
In allusion to the lack of a large number of fault data samples in machinery fault diagnosis, the thesis put forward a new method of machinery fault diagnosis based on support vector machine. And the principle and algorithm of the method has been introduced. Then the multi-fault classifier has been built using simulative fault data. This diagnostic method only need a small amount of time-domain fault data samples to train the fault classification, and don't need to extract the feature amount. In addition, we verify the correctness of this fault classifier through the application of fault classification in steam turbine generator set. The diagnostic method is simple and has strong ability of fault classification.
\end{abstract}

Keywords: support vector machine; machinery fault diagnosis; multi-fault classifier; hydroturbine generating units

\section{Introduction}

Industrial steam turbine is referred to as the "prime mover" in the industrial field. It is a key technical equipment. And it is widely used in various industrial fields [1, 2]. However, a series of fault occurs, such as bearing loose, rub, etc. These failures brought great losses to country and people. To avoid and reduce the occurrence of these failures, we should detect the security performance of steam turbine unit timely and effectively [3]. With the development of computer and artificial intelligence technology, and the combination of the mechanical equipment fault diagnosis and the intelligent diagnostic technology become a more important diagnostic technology [4].

In this paper, the support vector machine learning methods will be applied in hydropower generating unit vibration fault diagnosis field. And multi-fault classifier based on support vector machine $[5,6]$ is built. When fault samples are small and the characteristic quantities of data samples are not extracted, we can identify and classify the four common vibration fault of hydropower generating unit using analog fault samples directly. The effect is satisfactory.

\section{Basic principle of support vector machine classification}

Statistical learning theory is the statistical theory of small sample, and it research the statistical regularities and learning method of small samples.

Support vector machine [7] is a new machine learning method based on VC-dimension theory of statistical theory and minimization principle of structural risk, and it can solve the 
practical problems of nonlinearity and local minima. SVM is proposed by Vapnik in the 1990s. And since then it has been in a stage of rapid development. SVM can transform multidimensional space problem into low dimensional space problem. Therefore, when dealing with the multidimensional space problem, the optimal classification line can be taken for optimal classification plane. The SVM system has two aspects. The one aspect is training. In this step, the SVM model is obtained by training the sample data. The other aspet is discriminating. In this step, the other data is identified and classfied by the established SVM model. The General flowchart of SVM system is shown as Figure 1.

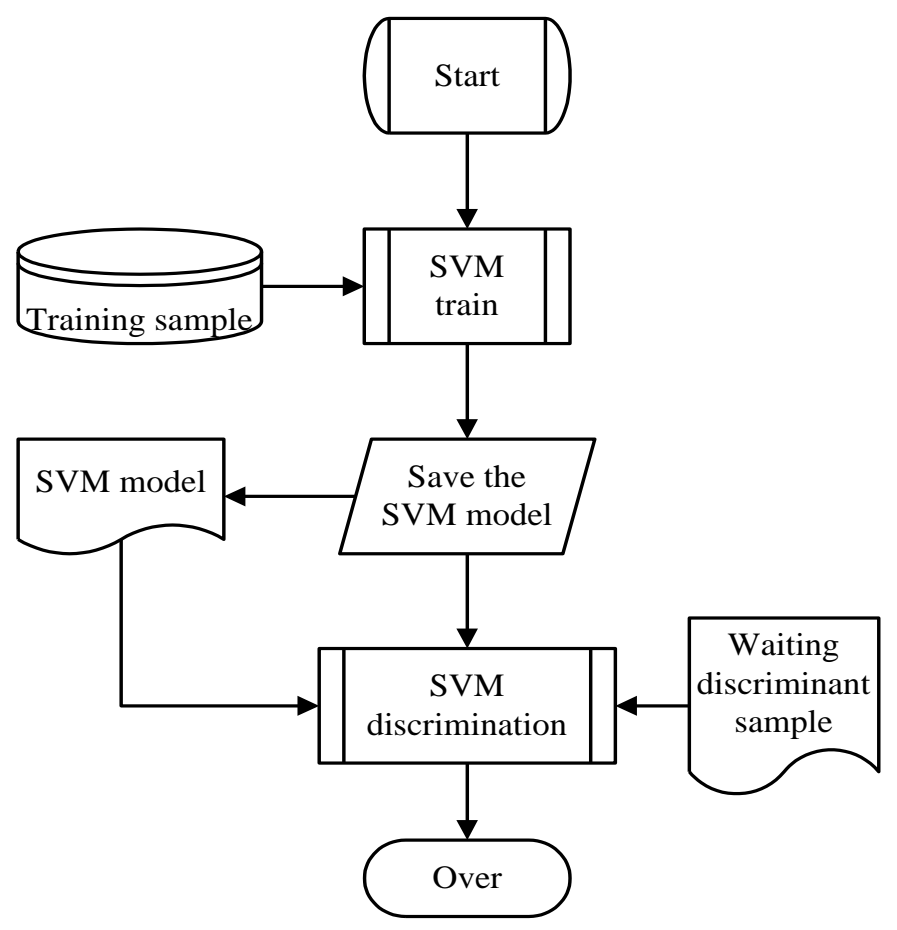

Figure 1. The General Flowchart of SVM System

If given two categories training data sample set is $\left(x_{i}, y_{i}\right), i=1,2, \cdots, n, \quad x \in R^{d}$, $y \in\{+1,-1\}$, can be separated by a hyperplane linear, the classification hyperplane equation is $w \cdot x+b=0 . n$ is the number of training samples. $d$ is the dimension of each training sample vector. $y$ is the category label. $w$ is the weight coefficient vector of classification surface. $b$ is classification field value.

For the classification of linear inseparable, we could transform sample input space into linear problem which is in a high-dimensional space through nonlinear transformation. If there are non-linear mapping, we can map the sample of the input space into the highdimensional feature space $H$ (maybe infinite dimensional). When you construct an optimal hyperplane in the feature space $H$, the training algorithm only use the dot product in the space (that is $<\Phi\left(x_{i}\right), \Phi\left(x_{j}\right)>$ ). According to the functional theory, As long as a nuclear function satisfies the Mercer condition, It corresponds to the inner product of a transformation space(that is $\left.K\left(x_{i}, x_{j}\right)=\Phi\left(x_{i}\right) \cdot \Phi\left(x_{j}\right)\right)$. So, we only need do the inner product operation in 
the high-dimensional space actually. And this inner product operation can be achieved using the original space function. And you needn't know the specific form of $\Phi(x)$.

Right now, the objective function is

$$
\begin{aligned}
& \max Q(a)=\sum_{i=1}^{n} a_{i}-\frac{1}{2} \sum_{i, j=1}^{n} a_{i} a_{j} y_{i} y_{j} K\left(x_{i}, x_{j}\right) \\
& \text { Subject to } \sum_{i=1}^{n} y_{i} a_{i}=0, \quad a_{i} \geq 0, \quad i=1,2, \cdots, n
\end{aligned}
$$

The general expressions of support vector machine classifier function is

$$
f(x)=\operatorname{sgn}\left\{\sum_{\text {SMM }} a_{i} y_{i} K\left(x_{i}, x\right)+\mathrm{b}\right\}
$$

The different support vector machine can be acquired by selecting different the inner product kernel function.

The above is mainly to solve binary classification problem based on support vector machine. Because there are many species failure of the turbine generator, we can solve the failure by multi-classification algorithm. Algorithm is as follows ${ }^{[8,9]}$.

(1) Construct two types of classification rules. One is $f_{k}(x)(k=1,2, \cdots, n)$. It could separate one training sample from other training samples. If the vector $x_{i}$ belongs to the $k$ class, $\operatorname{sgn}\left(f_{k}\left(x_{i}\right)\right)=1$. Otherwise, $\operatorname{sgn}\left(f_{k}\left(x_{i}\right)\right)=-1$.

(2) Construct an n-class classifier through selecting the category (m=argmax $\left.\left\{f_{1}\left(x_{i}\right), f_{2}\left(x_{i}\right), \ldots, f_{n}\left(x_{i}\right)\right\}\right)$ of corresponding to the maximum in the function $\left(f_{k}\left(x_{i}\right) \quad(i=1,2, \cdots, n)\right)$.

\section{The Choice of Kernel Function}

The Input vector $\mathrm{x}$ is mapped to a high dimensional feature space through some nonlinear mapping.Then the optimal separating hyperplane is built in this high-dimensional space.It is shown as Figure 2.

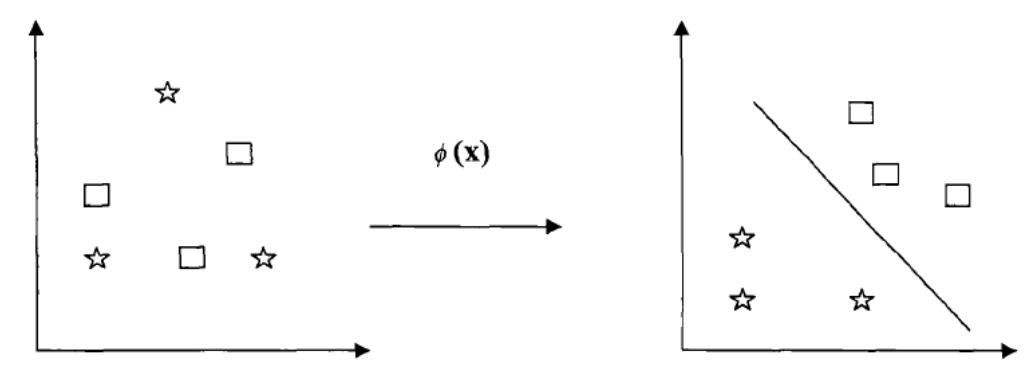

Figure 2. The Kernel Function

In classifying the sample,the inner product of high-dimensional space must be replaced by the kernel function. Support vector machine played functionality in classification problems. Selecting the kernel function, it must be accurate. It not only to be up to Mercer conditions, 
but also to understand the distribution of training samples. Feature space and kernel function has a very close relationship.The classifier along with kernel function is changed.

The following Mercer conditions: Arbitrary function $\varphi(x) \neq 0$ and Symmetric function $k(x, y)$ must meet the following conditions.

$$
\left\{\begin{array}{c}
\int_{-\infty}^{+\infty} \varphi^{2}(x) d x<0 \\
\iint_{-\infty}^{+\infty} k(x, y) \varphi(x) \varphi(y) d x d y>0
\end{array}\right.
$$

Gaussian radial basis kernel function (RBF) has four advantages. (1)To calculate, Its core value ranging from 0 to 1.(2)In classifying the sample, the sample number of the size have no effect with the classification results.(3)Kernel function can be viewed as an infinite number of polynomial kernel.(4)Parameters $\gamma$ only need to be adjusted.

\section{Fault Diagnosis of Steam Turbine Generator Set}

\subsection{The Establishment of Multi-Fault Classifier}

This fault diagnosis aims at steam turbine generator set of a thermal power plant. And the operating frequency of unit is $50 \mathrm{HZ}$. We take the three fault data samples of steam incentive, bearing loose and rubbing and normal data samples as training and test samples. The data sample is raw data of the time-domain and is directly collected from the unit high cylinder bearing seat ${ }^{[10]}$. And the frequency is $2000 \mathrm{HZ}$. The data length is 256 points. The waveforms of four data samples are shown in Figure 3.

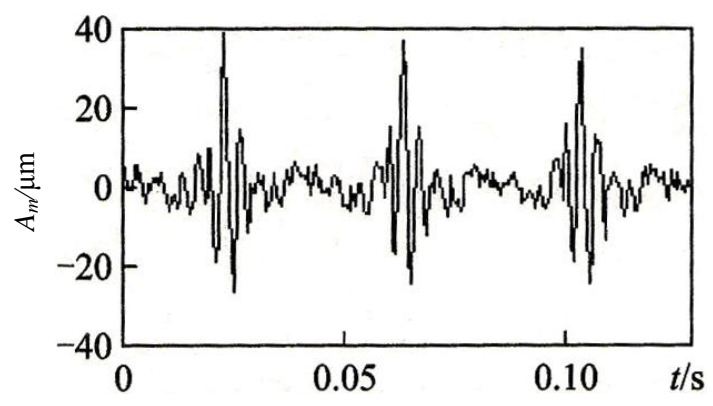

(a) Steam incentive fault data samples

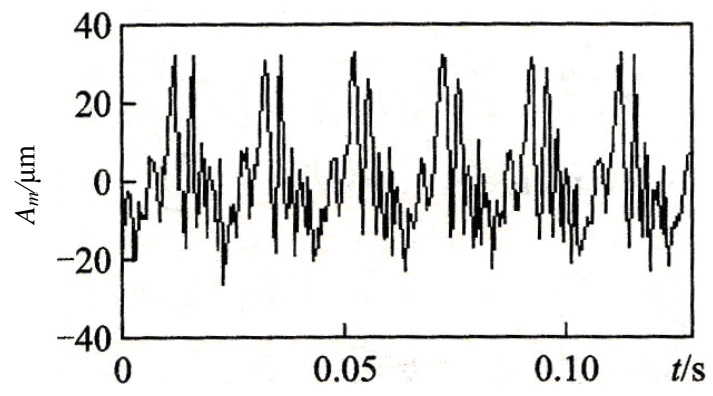

(b) Bearing loose fault data samples 


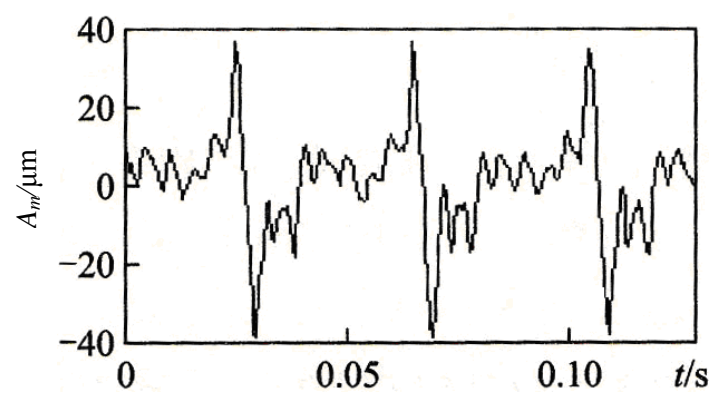

(c) Rubbing fault data samples

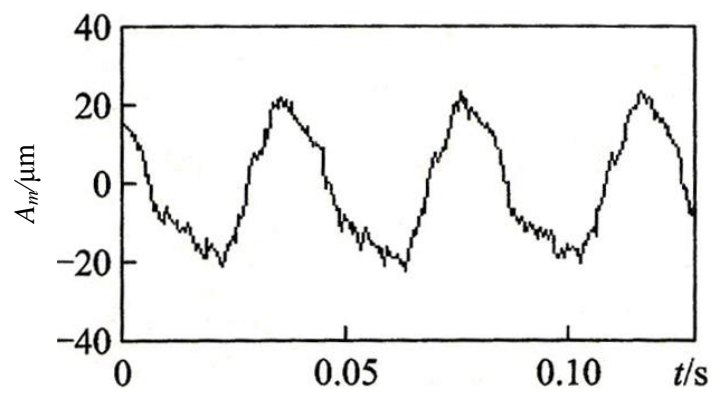

(d) Normal data samples

Figure 3. The Data Samples of Time-Domain Waveform

Ten data are selected as training samples from every data sample respectively. Then take the ten data from the normal data sample as one category that is marked as 1 . And take the other thirty data from the other data samples as the one category that is marked as -1 . Then the optimization coefficient $\alpha_{i}$ could be obtained according to formula (1). The form of kernel function $K\left(x_{i}, y_{i}\right)$ and the punishment coefficient should be determined in formula (1) and its constraint conditions. We should choose the polynomial kernel function $K\left(x_{i}, y_{i}\right)=\left(x_{i} \cdot y_{i}+1\right)^{2}$ and $\mathrm{C}=1000$ after optimizing. The training samples of corresponding to nonzero item are support vectors in $\alpha_{i}$ after getting $\alpha_{i}$. Four binary classifiers SVM1 SVM4 under the four states of the unit could be built according to discriminating formula (3). Each classifier can identify and isolate one fault from the four data samples. The training time is not more than $0.4 \mathrm{~s}$. If we combine the four binary classifiers as shown in Figure 4, the multi-fault classifer that can identify the four data samples is obtained.

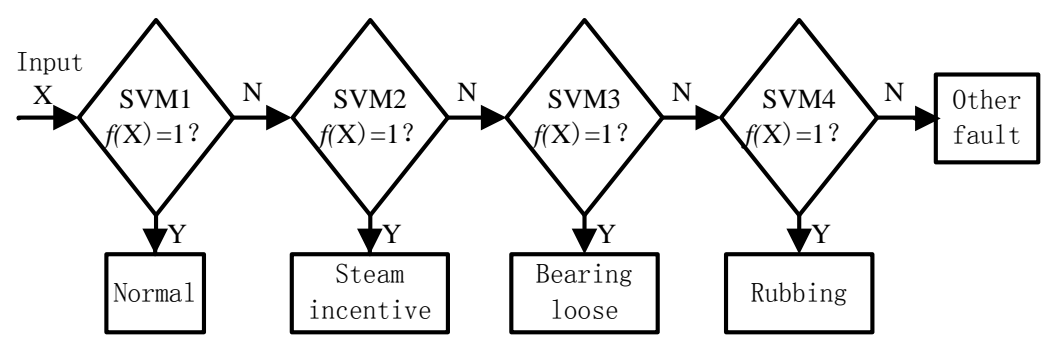

Figure 4. The Multi-Fault Classifer Flow Chart of Steam Turbine Generating Set 
Four binary classifiers SVM1 SVM4 can identify and isolate the normal, seam incentive, bearing loose and rubbing data samples as shown in Figure 4. In the classification testing, firstly, input the data $x$ to classifiers 1 . If discriminating formula $f(x)$ output 1 , the unit is normal and the testing is over. Or else, input $x$ to classifiers 2 . If discriminating formula $f(x)$ output 1,the unit has steam incentive fault and the testing is over. Or else, input $x$ to classifiers 3 . And so on, until the classifier 4 , if discriminating formula $f(x)$ doesn't output 1 , the unit maybe has other fault.

\subsection{The Testing Result}

In order to checking the effect of fault classification. We select 3 data respectively $\left(x_{1} \sim\right.$ $x_{12}$ ) from every test sample. The testing result is shown in Table 1 . The checking result coincide with the type of data sample. So, it indicates the classification can identify and isolate the fault.

Table 1. The testing result

\begin{tabular}{|c|c|c|c|c|c|c|}
\hline Test samples & $\begin{array}{c}\text { The type } \\
\text { of fault }\end{array}$ & SVM1 & SVM2 & SVM3 & SVM4 & Result \\
\hline $\mathbf{x 1} \sim \mathbf{x 3}$ & $\begin{array}{c}\text { Normal } \\
\text { data }\end{array}$ & 1 & & & & $\begin{array}{c}\text { Normal } \\
\text { data }\end{array}$ \\
\hline $\mathbf{x 4} \sim \mathbf{x 6}$ & $\begin{array}{c}\text { Steam } \\
\text { incentive }\end{array}$ & -1 & 1 & & & $\begin{array}{c}\text { Steam } \\
\text { incentive }\end{array}$ \\
\hline $\mathbf{x 7} \sim \mathbf{x 9}$ & $\begin{array}{c}\text { Bearing } \\
\text { loose }\end{array}$ & -1 & -1 & 1 & & $\begin{array}{c}\text { Bearing } \\
\text { loose }\end{array}$ \\
\hline $\mathbf{x 1 0} \sim \mathbf{x 1 2}$ & Rubbing & -1 & -1 & -1 & 1 & Rubbing \\
\hline
\end{tabular}

\section{Conclusions}

In this paper, the multi-fault classification based on support vector machine is established according to the statistical theory. This classifier only need a small amount of time-domain fault data samples to train the fault classification, and don't need to extract the feature amount. And the correctness of this fault classifier is verified through the application of fault classification in steam turbine generator set. The diagnostic method is simple and has strong ability of fault classification.

\section{Acknowledgements}

This work is financially supported by the 2012 postgraduate innovation scientific research fund project of Heilongjiang province (YJSCX2012-324HLJ)

\section{References}

[1] L. Shen, F. E. H. Tay and L. Qu, "Fault diagnosis using rough sets theory", Computers in Industr., vol. 1, (2000), pp. 43.

[2] F. E. H. Tay and L. Shen, "Fault diagnosis based on rough set theory", Engineering Application of Artificial Intelligenc., vol. 1, (2003), pp. 16.

[3] J. Fu, T. Zhang and Z. Ren, "Detection of Coal Mine Roof Based on Support Vector Machine Ensemble", Microcomputer \& Its Applications, vol. 17, (2012), pp. 31.

[4] Z. Zhang, L. Li and Z. He, "Research on Diagnosis Method of Machinery Fault Based on Support Vector Machine”, Journal of Xian Jiao Tong University, vol. 12, (2002), pp. 36. 
[5] V. Vapnik, "The Nature of Statistical Learning Theory", Springer-Verlag, New York, (1999).

[6] C. W. Hsu and C. J. Lin, "A comparison of methods for multi-class support vector machines", IEEE Trans on Neural Networks, vol. 2, (2002), pp. 13.

[7] S. Ding, B. Qi and H. Tan, "An Overview on Theory and Algorithm of Support Vector Machines", Journal of University of Electronic Science and Technology of China, vol. 1, (2011), pp. 40.

[8] C. Cortes and V. Vapni, "Support-vector network", Machine Learning, vol. 3, (1995), pp. 20.

[9] L. K. Hansen and P. Salamon, "Neural network ensemble", IEEE Trans on Pattern Analysis and Machine Intelligence, vol. 10, (1999), pp. 12.

[10] Z. He, J. Cheng and T. Wang, "Theories and Applications of Machinery Fault Diagnostics", Higher Education Press, BeiJing, (2010). 
International Journal of Hybrid Information Technology Vol.6, No.6 (2013) 